\title{
APPROXIMATING FIXED POINTS OF NONEXPANSIVE AND GENERALIZED NONEXPANSIVE MAPPINGS
}

\author{
M. MAITI and B. SAHA \\ Department of Mathematics \\ Indian Institute of Technology, Kharagpur, India
}

(Received May 20, 1991 and in revised form November 15, 1991)

\section{ABSTRACT.}

In this paper we consider a mapping $S$ of the form

$$
S=\alpha_{0} I+\alpha_{1} T+\alpha_{2} T^{2}+\ldots+\alpha_{k} T^{*},
$$

where $\alpha_{i} \geq 0 . \alpha_{1}>0$ with $\sum_{i=0}^{k} \alpha_{i}=1$, and show that in a uniformly convex Banach space the Picard iterates of $S$ converge to a fixed point of $T$ when $T$ is nonexpansive or generalized nonexpansive or even quasinonexpansive.

KEY WORDS AND PHRASES: Fixed points, nonexpansive mappings, uniformly convex Banach spaces. 1991 AMS SUBJECT CLASSIFICATION CODES. Primary 47H10; Secondary 54H25.

\section{INTRODUCTION.}

Let $B$ be a Banach space and $C$ a convex subset of $B$. A mapping $T: C \rightarrow C$ is said to be nonexpansive if $\|T x-T y\| \leq\|x-y\|$ for all $x, y \in C$. A mapping $T: C \rightarrow C$ is said to be quasi-nonexpansive if $T$ has a fixed point $p$ such that $\|T x-p\| \leq\|x-p\|$ for all $x \in C$. The concept of quasi-nonexpansiveness is more general than that of nonexpansiveness. Indeed, a nonexpansive mapping with at least one fixed point is quasi-nonexpansive, but there exists quasi-nonexpansive mappings which are not nonexpansive. See, for example, Petryshyn and Williamson [7].

If $T$ is nonexpansive, then the Picard iterates of $T$ may not converge and, even if they do converge, they may not converge to a fixed point of $T$. However, to circumvent the difficulty, one may consider the mapping

$$
T_{\lambda}=(1-\lambda) I+\lambda T,
$$

where $I$ is the identity mapping and $0<\lambda<1$, and show that the Picard iterates of $T_{\lambda}$ converge to a fixed point of $T$ under certain restrictions, see [3,5,10]. Generalizing the idea Kirk [6] has introduced a mapping $S$ given by

$$
S=\alpha_{0} I+\alpha_{1} T+\alpha_{2} T^{2}+\ldots+\alpha_{k} T^{*}
$$

where $\alpha_{i} \geq 0, \alpha_{1}>0$ with $\sum_{i=0}^{k} \alpha_{i}=1$, and has shown that the Picard iterates of $S$ converge to a fixed point of $T$ under conditions similar to those imposed in connection with the convergence of Picard iterates of $T_{\lambda}$.

Our purpose here is two-fold. First we show that the Picard iterates of $S$ converge to a fixed point of $T$ under conditions weaker than those imposed by Kirk [6]. Secondly, we establish that the Picard iterates of $S$ converge to a fixed point of $T$ even when $T$ is generalized nonexpansive, i.e., when $T$ satisfies 


$$
\|T x-T y\| \leq a\|x-y\|+b\{\|x-T x\|+\|y-T y\|\}+c\{\|y-T x\|+\|x-T y\|\}
$$

for all $x, y \in C$, where $a, b, c \geq 0$ with $a+2 b+2 c \leq 1$. Then the analysis has been extended to a more general mapping resulting in generalization of some results obtained by Ray and Rhoades [9].

\section{CONVERGENCE TO FIXED POINTS}

It has been established by Kirk [6] that $S$ and $T$ have common fixed points if $T$ is nonexpansive. Let the common fixed point set be denoted by $F$. Further, the set $F$ is closed when $T$ is nonexpansive or even when $T$ is quasi-nonexpansive (see Dotson [2]). We now state the following conditions:

CONDITION-A. A mapping $T: C \rightarrow C$ with a nonempty fixed point set $F$ is said to satisfy Condition-A if there is a nondecreasing function $f:[0, \infty) \rightarrow[0, \infty)$ with $f(0)-0$ and $f(r)>0$ for all $r \in(0, \infty)$ such that $\|x-S x\| \geq f(d(x, F))$ for all $x \in C$, where $d(x, F)=\inf _{p \in F}\|x-p\|$.

CONDITION-B. A mapping $T: C \rightarrow C$ with a nonempty fixed point set $F$ is said to satisfy Condition-B if there exists a number $\alpha>0$ such that $\|x-S x\| \geq \alpha d(x, F)$ for all $x \in C$.

It may be remarked that the mappings which satisfy Condition-B also satisfy Condition-A. However, Condition-B may be verified easily by giving examples. It may be further remarked that Conditions I and II of Senter and Dotson [11] are identical with Conditions A and B when $\alpha_{2}=\alpha_{3}=\ldots=\alpha_{k}=0$.

We now recall the following lemma due to Dotson [1]. This will be used later to establish our results.

LEMMA. If the sequences $\left\{s_{n}\right\}$ and $\left\{t_{n}\right\}$ are in the closed unit ball of a uniformly convex Banach space and $\left\{z_{n}\right\}=\left\{\left(1-\alpha_{n}\right) s_{n}+\alpha_{n} t_{n}\right\}$ satisfies $\lim _{n \rightarrow \infty}\left\|z_{n}\right\|=1$, where $0<a \leq \alpha_{n} \leq b<1$, then $\lim _{n \rightarrow \infty}\left\|s_{n}-t_{n}\right\|=0$.

THEOREM 1. Let $C$ be a nonempty, closed, convex and bounded subset of a uniformly convex Banach space $B$ and $T: C \rightarrow C$ be a nonexpansive mapping. If $T$ satisfies Condition-A, where $F$ is the fixed point set of $T$ in $C$, then for an arbitrary $x_{0} \in C$, the Picard iterates $\left(S^{n} x_{0}\right)$ converge to a member of $F$.

PROOF. If $x_{0} \in F$, then the result is trivial. We assume that $x_{0} \in C-F$. Then, setting $x_{n}-S^{n} x_{0}$, we have for an arbitrary $p \in F$

$$
\begin{aligned}
\left\|x_{n+1}-p\right\|=\| S^{n+1} x_{0} & -p\|-\| S x_{n}-p \| \\
& =\left\|\alpha_{0} x_{n}+\alpha_{1} T x_{n}+\alpha_{2} T^{2} x_{n}+\ldots+\alpha_{k} T^{k} x_{n}-p\right\| \\
& =\| \alpha_{0}\left(x_{n}-p\right)+\alpha_{1}\left(T x_{n}-p\right)+\alpha_{2}\left(T^{2} x_{n}-p\right)+\ldots+\alpha_{k}\left(T^{k} x_{n}-p \|\right. \\
& \leq \alpha_{0}\left\|x_{n}-p\right\|+\alpha_{1}\left\|T x_{n}-p\right\|+\alpha_{2}\left\|T^{2} x_{n}-p\right\|+\ldots+\alpha_{k}\left\|\left(T^{k} x_{n}-p\right)\right\| \\
& \leq\left\|x_{n}-p\right\| .
\end{aligned}
$$

This implies that $d\left(x_{n+1}, F\right) \leq d\left(x_{n}, F\right)$ and hence that the sequence $\left\{d\left(x_{n}, F\right)\right\}$ is nonincreasing. Then $\lim _{n \rightarrow \infty} d\left(x_{n}, F\right)$ exists. In the sequel we shall show that this limit is zero.

Suppose that $\lim _{n \rightarrow \infty} d\left(x_{n}, F\right)=b>0$. Then, for a $p \in F, \lim _{n \rightarrow \infty}\left\|x_{n}-p\right\|-b^{\prime} \geq b>0$. Choose a positive integer $N$ such that $\left\|x_{n}-p\right\| \leq 2 b^{\prime}$ for $n \geq N$. Set $y_{n}^{i}=\left(T^{i} x_{n}-p\right)\left\|x_{n}-p\right\|$ for all $n$ and all $i=0,1,2, \ldots, k$ with $T^{0} x_{n}=x_{n}$. Then $\left\|y_{n}^{i}\right\| \leq 1$. Further, set $z_{n}=\alpha_{0} y_{n}^{0}+\left(1-\alpha_{0}\right) t_{n}$, where $t_{n}=\sum_{i=1}^{k}\left(\alpha_{i} y_{n}^{i}\right) /\left(1-\alpha_{0}\right)$ with $\left\|t_{n}\right\| \leq 1$. Then $\left\{y_{n}^{0}\right\}$ and $\left\{T_{n}\right\}$ are in the closed unit ball. Now 


$$
\begin{aligned}
\left\|z_{n}\right\| & =\left\|\alpha_{0} \frac{x_{n}-p}{\left\|x_{n}-p\right\|}+\alpha_{1} \frac{T x_{n}-p}{\left\|x_{n}-p\right\|}+\alpha_{2} \frac{T^{2} x_{n}-p}{\left\|x_{n}-p\right\|}+\ldots+\alpha_{k} \frac{T^{*} x_{n}-p}{\left\|x_{n}-p\right\|}\right\| \\
& =\frac{\left\|\alpha_{0} x_{n}+\alpha_{1} T x_{n}+\alpha_{2} T^{2} x_{n}+\ldots+\alpha_{k} T^{*} x_{n}-p\right\|}{\left\|x_{n}-p\right\|}=\frac{\left\|x_{n+1}-p\right\|}{\left\|x_{n}-p\right\|}
\end{aligned}
$$

implying $\left\|z_{n}\right\| \rightarrow 1$ as $n \rightarrow \infty$. But, for $n \geq N$, we have

$$
\begin{aligned}
\left\|y_{n}^{0}-t_{n}\right\| & =\left|\frac{x_{n}-p}{\left\|x_{n}-p\right\|}-\frac{1}{\left(1-\alpha_{0}\right)_{i}} \sum_{i=1}^{k} \frac{\alpha_{i}\left(T^{i} x_{n}-p\right)}{\left\|x_{n}-p\right\|}\right| \\
& =\left|\frac{x_{n}-p}{\left\|x_{n}-p\right\|}-\frac{S x_{n}-\alpha_{0} x_{n}-\left(1-\alpha_{0}\right) p}{\left(1-\alpha_{0}\right)\left\|x_{n}-p\right\|}\right| \\
& =\frac{\left\|x_{n}-S x_{n}\right\|}{\left(1-\alpha_{0}\right)\left\|x_{n}-p\right\|} \\
& \geq \frac{f\left(d\left(x_{n}, F\right)\right)}{\left(1-\alpha_{0}\right)\left\|x_{n}-p\right\|} \geq \frac{f(b)}{2 b^{\prime}\left(1-\alpha_{0}\right)}>0,
\end{aligned}
$$

implying $\lim _{n \rightarrow \infty}\left\|y_{n}^{0}-t_{n}\right\| \neq 0$, which contradicts the lemma. Hence $\lim _{n \rightarrow \infty} d\left(x_{n}, F\right)-0$. This implies that $\left\{x_{n}\right\}$ converges to a member of $F$, since $F$ is closed.

REMARK 1. It is obvious that the above theorem holds if Condition-B is satisfied instead of Condition-A.

REMARK 2. Condition-A is more general than the condition imposed by Kirk [6] in establishing the convergence of Picard iterates $\left\{S^{n} x_{0}\right\}$, see Senter and Dotson [11].

REMARK 3. In the above theorem the existence of a nonempty fixed point set $F$ is not assumed and is ensured by the conditions assumed therein. However, if we assume that $T$ has a nonempty fixed point set $F$, then $T$ need not be assumed to be nonexpansive and it is enough for $T$ to be quasi-nonexpansive. Further, $C$ need not be bounded. Indeed, the following result holds.

THEOREM 2. Let $C$ be a nonempty, closed and convex subset of a uniformly convex Banach space $B$ and $T: C \rightarrow C$ be a quasi-nonexpansive mapping. If $T$ satisfies Condition-A, where $F$ is the fixed point set of $T$ in $C$, then, for an arbitrary $x_{0} \in C$, the Picard iterates $\left\{S^{n} x_{0}\right\}$ converge to a member of $F$.

The proof may be established exactly in the same way as in Theorem 1. It only remains to be shown here that $S$ and $T$ have common fixed points. A fixed point of $T$ is obviously a fixed point of $S$. We now show that the converse is also so. Let $p$ be a fixed point of $S$. Then from Condition-A it is obvious that $d(p, F)=0$, implying $p \in \bar{F}$. Since $T$ is quasi-nonexpansive, $F$ is closed and hence $p \in F$, i.e., $p$ is a fixed point of $T$. Hence the result.

Next, we show that the Picard iterates of $S$ converge to a fixed point of $T$ even when $T$ is generalized nonexpansive. However, one need not assume Condition-A or Condition-B in this case. These conditions are automatically satisfied.

THEOREM 3. Let $C$ be a nonempty, bounded, closed and convex subset of a uniformly convex Banach space $B$ and $T: C \rightarrow C$ be a continuous mapping such that

$$
\|T x-T y\| \leq a\|x-y\|+b\{\|x-T x\|+\|y-T y\|\}+c\{\|x-T y\|+\|y-T x\|\}
$$

for all $x, y \in C$, where $a, c \geq 0$ and $b>0$ with $a+2 b+2 c \leq 1$. Then for an arbitrary $x_{0} \in C$, the Picard iterates $\left\{S^{n} x_{0}\right\}$ converges to the unique fixed point of $T$.

PROOF. By Theorem 2 of Goebel, Kirk and Shimi [4] the mapping $T$ has a unique fixed point $p$, say. Setting $y=p$ in (2.3) we have 


$$
\begin{aligned}
\|T x-p\| & \leq(a+c)\|x-p\|+b\|x-T x\|+c\|T x-p\| \\
& \leq(a+b+c)\|x-p\|+(b+c)\|T x-p\|,
\end{aligned}
$$

implying

$$
\|T x-p\| \leq \frac{a+b+c}{1-b-c}\|x-p\| \leq\|x-p\|,
$$

since $a+2 b+2 c \leq 1$. Thus $T$ is quasi-nonexpansive. Further, it is easy to verify that

$$
\|S x-p\| \leq\|T x-p\| \leq\|x-p\|,
$$

implying $S$ is also quasi-nonexpansive.

It is obvious that $p$ is also a fixed point of $S$. We now show that $S$ cannot have a fixed point other than $p$. If possible, let $q(\neq p)$ be a fixed point of $S$. Then

$$
\begin{aligned}
\|q-T q\| & =\|S q-T q\| \\
& =\left\|\alpha_{0} q+\alpha_{1} T q+\alpha_{2} T^{2} q+\ldots+\alpha_{k} T^{k} q-T q\right\| \\
& =\left\|\alpha_{0}(q-T q)+\alpha_{2}\left(T^{2} q-T q\right)+\ldots+\alpha_{k}\left(T^{k} q-T q\right)\right\| \\
& \leq \alpha_{0}\|q-T q\|+\alpha_{2}\left\|T^{2} q-T q\right\|+\ldots+\alpha_{k}\left\|T^{k} q-T q\right\| \\
& \leq \alpha_{0}\{\|q-p\|+\|T q-p\|\}+\alpha_{2}\left\{T^{2} q-p\|+\| T q-p \|\right\} \\
& \quad+\ldots+\alpha_{k}\left\{\left\|T^{k} q-p\right\|+\|T q-p\|\right\} \\
& \leq 2\left(\alpha_{0}+\alpha_{2}+\ldots+\alpha_{k}\right)\|q-p\|-2\left(1-\alpha_{1}\right)\|q-p\| .
\end{aligned}
$$

Since $T$ is generalized nonexpansive, we have

$$
\begin{aligned}
\|T q-p\| & =\|T q-T p\| \\
& \leq a\|q-p\|+b\|T q-q\|+c\{\|T q-p\|+\|q-p\|\} \\
& \leq(a+2 c)\|q-p\|+b \mid T q-q \| .
\end{aligned}
$$

Substituting from (2.6) into (2.7) and noting that $a+2 c \leq 1-2 b$ we obtain

$$
\begin{aligned}
\|T q-p\| & \leq(1-2 b)\|q-p\|+2 b\left(1-\alpha_{1}\right)\|q-p\| \\
& =\|q-p\|-2 b \alpha_{1}\|q-p\| \\
& <\|q-p\|-2 b \alpha_{1}\|T q-p\|,
\end{aligned}
$$

implying

$$
\|T q-p\| \leq \frac{1}{1+2 b \alpha_{1}}\|q-p\|
$$

Now from (2.8) we have

$$
\|q-p\|=\|S q-p\| \leq\|T q-p\| \leq \frac{1}{1+2 b \alpha_{1}}\|q-p\|,
$$

which implies $q=p$, since $b, \alpha_{1}>0$. Thus $S$ and $T$ have a unique fixed point $p$.

Next, we show that $T$ satisfies Condition-B. For $x \in C$ we have

$$
\|T x-p\|=\|T x-T p\| \leq a\|x-p\|+b\|x-T x\|+c\{\|x-p\|+\|T x-p\|\},
$$

implying

$$
\|T x-p\| \leq \frac{a+c}{1-c}\|x-p\|+\frac{b}{1-c}\|x-T x\| .
$$


Now,

$$
\begin{aligned}
\|T x-x\| & \leq\|S x-T x\|+\|S x-x\| \\
& \leq \alpha_{0}\|x-T x\|+\alpha_{2}\left\|T^{2} x-T x\right\|+\ldots+\alpha_{k}\left\|T^{\star} x-T x\right\|+\|S x-x\| \\
& \leq 2\left(1-\alpha_{1}\right)\|x-p\|+\|S x-x\| .
\end{aligned}
$$

Also we observe that

$$
\|S x-p\| \leq \alpha_{0}\|x-p\|+\left(\alpha_{1}+\alpha_{2}+\ldots+\alpha_{k}\right)\|T x-p\|
$$

and that

$$
\|S x-x\| \geq\|x-p\|-\|S x-p\| .
$$

Now, substituting from (2.12) into (2.13) we derive

$$
\begin{aligned}
\|S x-x\| & \geq\|x-p\|-\alpha_{0}\|x-p\|-\left(\alpha_{1}+\alpha_{2}+\ldots+\alpha_{k}\right)\|T x-p\| \\
& =\left(1-\alpha_{0}\right)\{\|x-p\|-\|T x-p\|\},
\end{aligned}
$$

whence, using (2.10), we obtain

$$
\begin{aligned}
\|S x-x\| & \geq\left(1-\alpha_{0}\right)\left\{\|x-p\|-\frac{a+c}{1-c}\|x-p\|-\frac{b}{1-c}\|x-T x\|\right\} \\
& =\left(1-\alpha_{0}\right)\left\{\frac{1-a-2 c}{1-c}\|x-p\|-\frac{b}{1-c}\|x-T x\|\right\} \\
& \geq\left(1-\alpha_{0}\right)\left\{\frac{2 b}{1-c}\|x-p\|-\frac{b}{1-c}\|x-T x\|\right\} \\
& =\frac{b\left(1-\alpha_{0}\right)}{1-c}\{2\|x-p\|-\|x-T x\|\} .
\end{aligned}
$$

Now, substituting from (2.11) into (2.14) we get

$$
\begin{aligned}
\|S x-x\| & \geq \frac{b\left(1-\alpha_{0}\right)}{1-c}\left\{2\|x-p\|-2\left(1-\alpha_{1}\right)\|x-p\|-\|S x-x\|\right\} \\
& =\frac{b\left(1-\alpha_{0}\right)}{1-c}\left\{2 \alpha_{1}\|x-p\|-\|S x-x\|\right\},
\end{aligned}
$$

implying

$$
\|S x-x\| \geq \alpha\|x-p\|
$$

where

$$
\alpha=\frac{2 b \alpha_{1}\left(1-\alpha_{0}\right)}{1-c+b\left(1-\alpha_{0}\right)}>0,
$$

since $b, \alpha_{1}>0$. Thus $T$ satisfies Condition-B. Hence, by Theorem 2 , the result follows.

REMARK 4. It may be noted that the stipulation $\alpha_{1}>0$ in $S$ is necessary to rule out the possibility that fixed point of $S$ is a point at which $T$ may be periodic.

REMARK 5. If we do not restrict $b>0$ in Theorem 3 , then the fixed pint set of $T$ is not a singleton, and Condition-A is to be imposed to ensure the convergence of $\left\{S^{n} x_{0}\right\}$.

The present analysis can be extended to a more general mapping $T$ which satisfies

$$
\|T x-T y\| \leq \max \{\|x-y\|,[\|x-T x\|+\|y-T y\|] / 2,[\|y-T x\|+\|x-T y\|] / 2\}
$$

for all $x, \in C$. This mapping includes nonexpansive and generalized nonexpansive mappings (see Rhoades [8]). It is easy to verify that $T$ is quasi-nonexpansive. It has been proved by Ray and Rhoades [9] that $S$ and $T$ have the same fixed point set. Further, they have established the following theorem in this connection. 
THEOREM 4. ([9, Theorem 2]). Let $C$ be a nonempty, closed convex and bounded subset of a uniformly convex Banach space $B$ and $T$ a self-mapping of $C$ which satisfies (2.16). If $I-S$ maps bounded closed subsets of $C$ into closed sets of $B$, then, for each $x_{0} \in C$, the sequence $\left\{S^{n} x_{0}\right\}$ converges to a fixed point of $T$ in $C$.

However, the fact that $I-S$ maps bounded closed subsets of $C$ into closed sets implies Condition-A (see Senter and Dotson [11]). Thus Condition-A is more general and incorporating this condition we may obtain the following as generalizations of Theorems 2 and 3 of Ray and Rhoades [9].

THEOREM 5. Let $C$ be a nonempty, closed and convex subset of a uniformly convex Banach space $B$ and $T$ a self-mapping of $C$ which satisfies (2.16). If $T$ satisfies Condition-A, where $F$ is the nonempty fixed point set of $T$ in $C$, then, for an arbitrary $x_{0} \in C$, the Picard iterates $\left\{S^{n} x_{0}\right\}$ converge to a member of $F$.

We may note that $C$ need not be bounded in Theorem 5. Because we have assumed the existence of nonempty fixed point set of $T$ and Condition-A (see [11]). But the boundedness of $C$ cannot be omitted from the statement of Theorem 4.

ACKNOWLEDGEMENT. The authors are indebted to Professor B. E. Rhoades for his valuable suggestions for the improvement of the paper.

\section{REFERENCES}

[1] DOTSON, JR., W. G. On the Mann iterative process, Trans. Amer. Math. Soc. 149 (1970), 65-73.

[2] DOTSON, JR., W. G. Fixed points of quasi-nonexpansive mappings, J.Austral. Math. Soc. 13 (1972), 167-170.

[3] EDELSTEIN, M. A remark on a theorem of M. A. Krasnoselskii, Amer. Math. Monthly 73 (1966), 509-510.

[4] GOEBEL, K., KIRK, W. A. and SHIMI, T. N. A fixed point theorem in uniformly convex Banach spaces, Boll. Un. Mat. Ital. 7 (1973), 67-75.

[5] KRASNOSELSKII, M. A. Two remarks about the method of successive approximations, Uspehi Mat. Nauk 10 (1955), 123-127.

[6] KIRK, W. A. On successive approximations for nonexpansive mappings in Banach spaces, Glasgow Math. Jour. 12 (1971), 6-9.

[7] PETRYSHYN, W. V. and WILLIAMSON, JR., T. E. Strong and weak convergence of the sequence of successive approximations for quasi-nonexpansive mappings, J.Math. Anal. and Appl. 43 (1973), 459-497.

[8] RHOADES, B. E. A comparison of various definitions of contractive mappings, Trans. Amer. Math. Sce. 226 (1977), 257-290.

[9] RAY, B. K. and RHOADES, B. E. A class of fixed point theorems, Math. Semi. Notes 7 (1979), 477-489.

[10] SCHAEFER, H. Ober Die Methode seksessiver Approximation, Jber. Deutsch. Math. Verein, 59 (1957), 131-140.

[11] SENTER, H. F. and DOTSON, JR., W. G. Approximating fixed points of nonexpansive mappings, Proc. Amer. Math. Sec. 44 (1974), 375-380. 


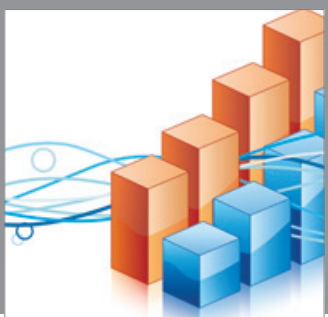

Advances in

Operations Research

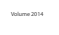

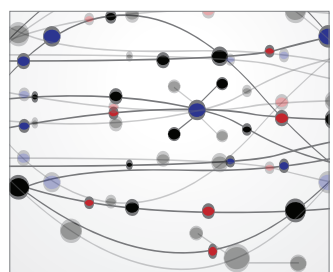

\section{The Scientific} World Journal
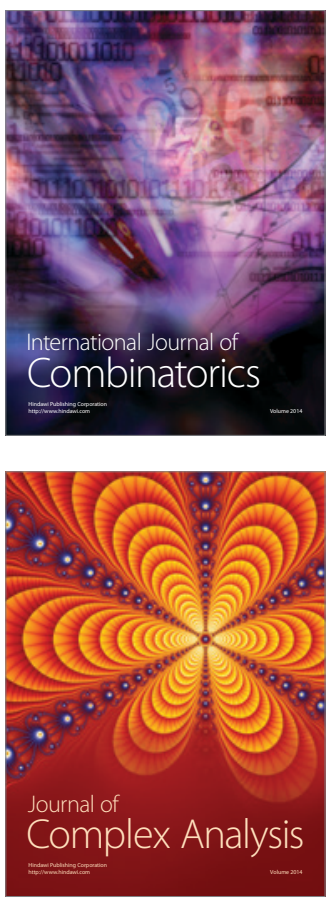

International Journal of

Mathematics and

Mathematical

Sciences
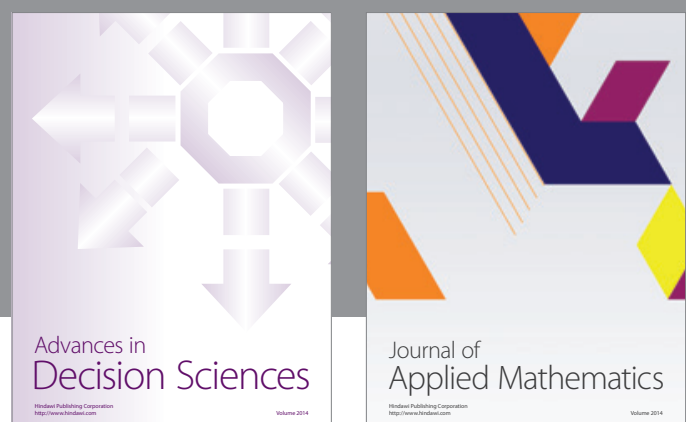

Journal of

Applied Mathematics
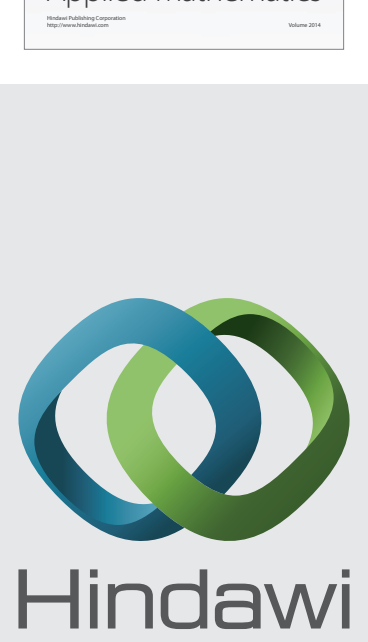

Submit your manuscripts at http://www.hindawi.com
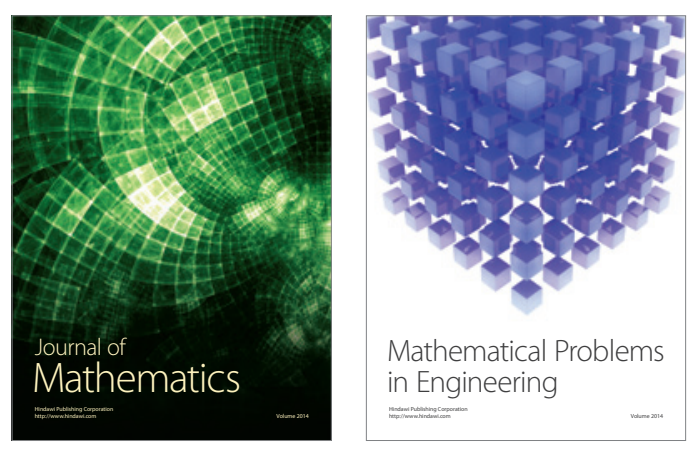

Mathematical Problems in Engineering
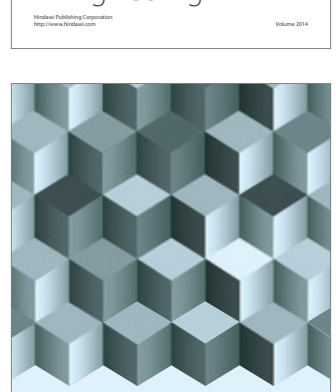

Journal of

Function Spaces
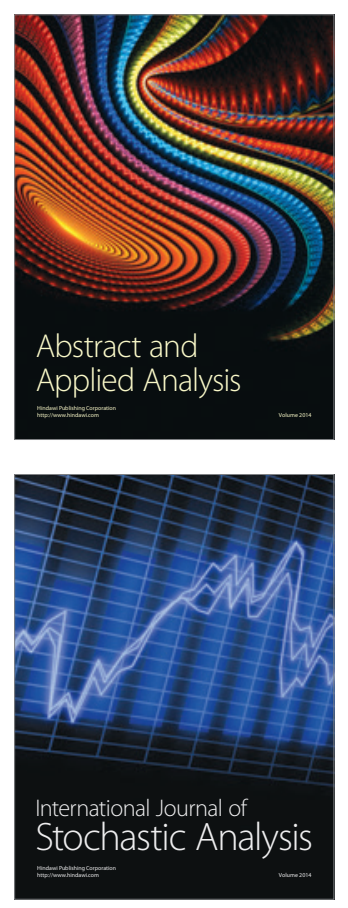

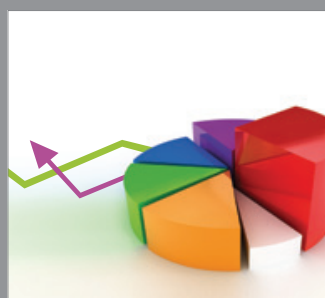

ournal of

Probability and Statistics

Promensencen
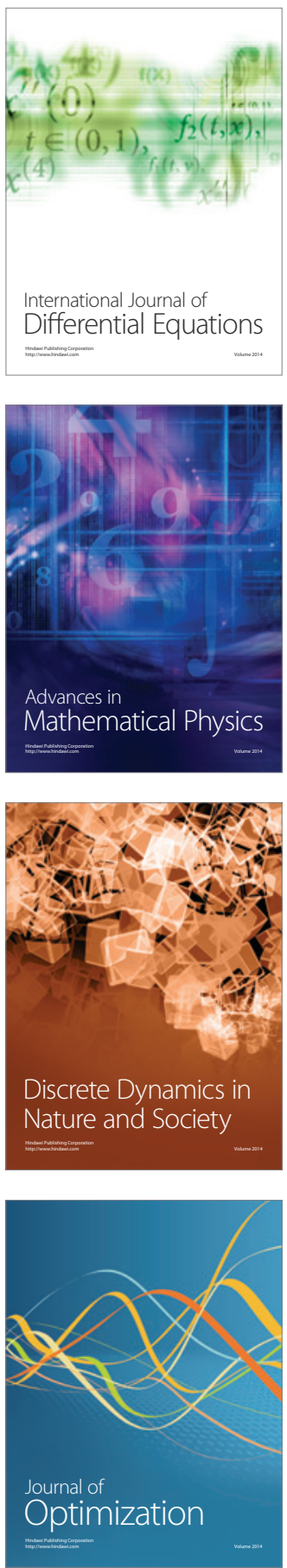